\title{
Muon g-2 and LHC phenomenology in the $L_{\mu}-L_{\tau}$ gauge symmetric model
}

\author{
Kazuhiro Tobe* ${ }^{* i}$ \\ Nagoya University \\ E-mail: tobedeken.phys.nagoya-u.ac.jp
}

\begin{abstract}
An anomaly of the muon anomalous magnetic moment (muon g-2) has been reported. The discrepancies between the Standard Model (SM) prediction and the measured value are about 3-4 $\sigma$. If this anomaly is due to new physics beyond the SM, we expect new particles and interactions related with the muon sector. In this talk, we consider a model with an $L_{\mu}-L_{\tau}$ gauge symmetry. Since the muon couples to the $L_{\mu}-L_{\tau}$ gauge boson (called Z" boson), its contribution to the muon g-2 can account for the discrepancy. On the other hand, the $\mathrm{Z}$ " boson does not interact with the electron nor quarks, and hence there are no strong constraints from collider experiments even if the Z" boson mass is of the order of the electroweak scale. We show an allowed region of a parameter space in the model, taking into account consistency with the electroweak precision measurements as well as the muon g-2. We study the Large Hadron Collider (LHC) phenomenology, and show that the current and future data would probe the interesting parameter space for this model.
\end{abstract}

KMI International Symposium 2013 on "Quest for the Origin of Particles and the Universe", 11-13 December, 2013

Nagoya University, Japan

\footnotetext{
* Speaker.

${ }^{\dagger}$ This talk is based on work in the collaboration with K. Harigaya, T. Igari, M. M. Nojiri, and M. Takeuchi in Ref. [1].
} 


\section{Introduction}

The standard model (SM) of elementary particles has been very successful to describe physics phenomena below the electroweak scale. A recent discovery of Higgs boson further strengthens the success of the SM. So far, no direct evidence of new physics has been discovered at the Large Hadron Collider (LHC).

Despite the remarkable success of the SM, several groups have reported an anomaly of muon anomalous magnetic moment $a_{\mu}=\left(g_{\mu}-2\right) / 2$ (moun g-2). The discrepancies between the SM prediction and the measured value of muon g-2 [2] are about $3-4 \sigma$ [3]. Therefore, the anomaly would be a good hint of new physics beyond the SM.

Since the size of the anomaly of muon $\mathrm{g}-2$ is of the same order as the electroweak contribution $a_{\mu}^{\mathrm{EW}}=(15.4 \pm 0.1) \times 10^{-10}[4]$ in the SM, we expect that the mass scale of new physics is of the order of the electroweak scale if the new coupling to muon is of the order of the electroweak gauge coupling. Such new particles with couplings to muon would be a good target at the LHC. Therefore, the phenomenological study for the LHC experiment is important to probe the origin of the anomaly of muon $\mathrm{g}-2$.

So far, many theoretical possibilities have been discussed to accommodate the anomaly of muon g-2. In order to explain the anomaly of muon g-2 by the new physics, muons have to have new interaction with new particles. The new interaction can be a Yukawa-type interaction. A good example is a supersymmetric extension of the SM. [5]. Other examples of this type have been discussed in Refs. [6], for example.

The new interaction can be a gauge interaction. Historically, gauge interactions have played many important roles on the elementary particle physics, and hence here we consider a gauge extension of the SM. If the gauge interaction is a flavor-universal type, constraints from collider experiments are very strong. Thus the gauge coupling should be very weak to avoid the constraints. In order to explain the anomaly of muon g-2 with the very weak gauge coupling, the mass of the extra gauge boson should be very small. Such a possibility has been discussed, and for example, the dark photon model [7] is one of interesting examples. If the gauge interaction is not flavor universal, the constraints may be weaker. One of such possibilities is $L_{\mu}-L_{\tau}$ gauge symmetric model [8]. In this talk, we would like to consider this model. We call it as " $Z$ " model" for simplicity.

\section{2. $L_{\mu}-L_{\tau}$ gauge symmetric model $\left(Z^{\prime \prime}\right.$ model)}

It has been known that a difference between individual lepton flavor number $L_{i}(i=e, \mu, \tau)$ is anomaly free without an addition of any exotic fermions in the SM. Therefore, an $\mathrm{U}(1)_{L_{i}-L_{j}}$ $(i \neq j)$ gauge symmetric model is one of the simplest and most economical extensions of the SM. In order to explain the anomaly of muon g-2 as well as to avoid the strong constraints from the collider experiments, the $\mathrm{U}(1)_{L_{\mu}-L_{\tau}}$ gauge symmetry is a very attractive possibility. The $\mathrm{U}(1)_{L_{\mu}-L_{\tau}}$ charges of both left- and right-handed muons (taus) and their neutrino partners $v_{\mu}\left(v_{\tau}\right)$ are +1 $(-1)$, and those of other particles in the SM are zero. Since the $\mathrm{U}(1)_{L_{\mu}-L_{\tau}}$ gauge boson $\left(Z^{\prime \prime}\right.$ gauge boson) does not couple to electron nor quarks, there are no strong constraints from the collider experiments. On the other hand, the $Z^{\prime \prime}$ boson can couple to muon, and hence it induces new contribution to muon $\mathrm{g}-2$, so that it can explain the anomaly of muon g-2. In next section, we show 
an interesting parameter space which accommodates the anomaly of muon g-2 as well as satisfies constraints from the electroweak observables.

\section{Contribution to muon $\mathrm{g}-2$ and electroweak precision observables}

The $Z^{\prime \prime}$ boson contributes to muon g-2 via a Feynman diagram shown in Fig. 1. The $Z^{\prime \prime}$ contribution $\delta a_{\mu}$ is shown in Fig. 2(a) as a function of the $Z^{\prime \prime}$ mass $m_{Z^{\prime \prime}}$ and the gauge coupling $g_{Z^{\prime \prime}}$. As can be seen from Fig. 2(a), the region with $g_{Z^{\prime \prime}}=O(1)$ and $m_{Z^{\prime \prime}}=O(100) \mathrm{GeV}$ is favored

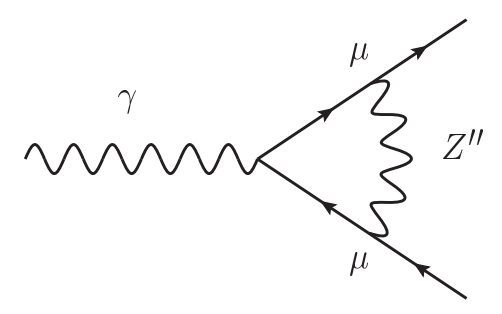

Figure 1: Feynman diagram for muon g-2, mediated by the $Z^{\prime \prime}$ gauge boson.

as we expected.

Since the relatively light $Z^{\prime \prime}$ boson is favored, it might significantly contribute to electroweak (EW) observables. If $\gamma$ in the diagram shown in Fig. 1 is replaced by $Z$, it contributes to $Z \mu \bar{\mu}$ vertex. When the gauge coupling $g_{Z^{\prime \prime}}$ is close to or larger than one, we find that the vertex correction is not negligible and it makes differences between the measured values and the theoretical predictions of $R_{\mu}$ and $\Gamma_{Z}$ larger.

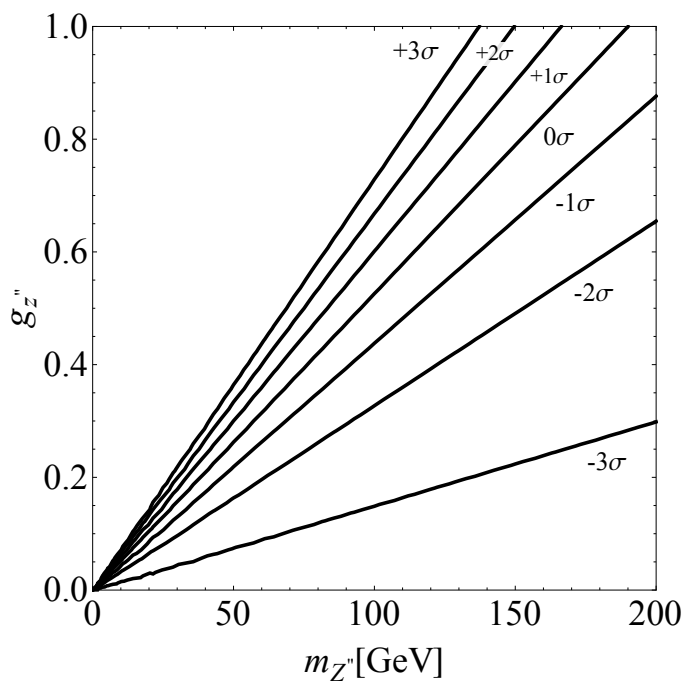

(a)

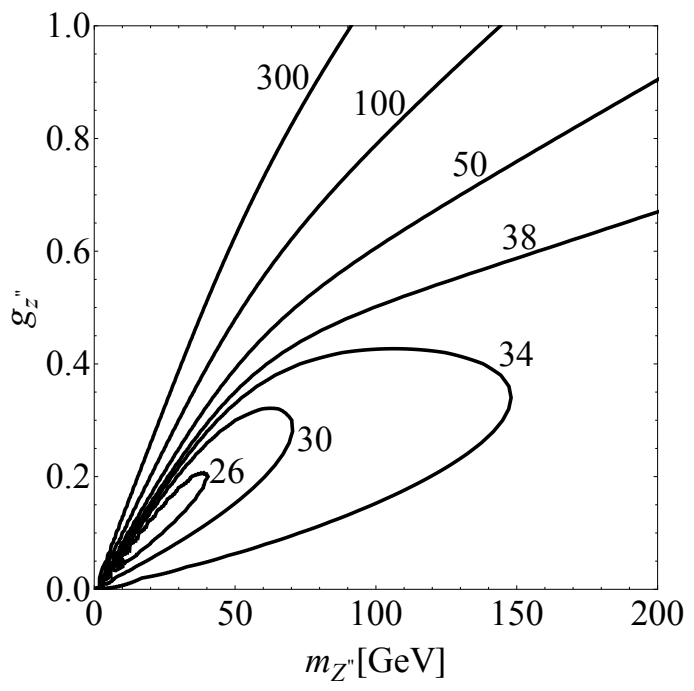

(b)

Figure 2: (a) Contours of the standard deviations for muon g-2 with the $Z^{\prime \prime}$ contribution $\left(\delta a_{\mu}\right)$ in $\left(m_{Z^{\prime \prime}}, g_{Z^{\prime \prime}}\right)$ plane. (b) The total $\chi^{2}$ in the $\left(m_{Z^{\prime \prime}}, g_{Z^{\prime \prime}}\right)$ plane. The $\chi^{2} /($ d.o.f $)$ of the SM is $35.1 /(22)$. The figures are taken from Ref. [1]. 
We calculate $\chi^{2}$ of the EW observables, $\Gamma_{Z}, \sigma_{h}^{0}, R_{e, \mu, \tau}, A_{\mathrm{FB}}^{0,(e, \mu, \tau)}, A_{e, \mu, \tau}, R_{b, c}, A_{\mathrm{FB}}^{0,(b, c)}, A_{b, c}$, $M_{W}, \Gamma_{W}, a_{\mu}, \Delta \alpha_{\text {had }}^{(5)}\left(M_{Z}^{2}\right), m_{t}$ and $m_{h}$ (see Ref. [1] for detail) and identify a favored region of $m_{Z^{\prime \prime}}$ and $g_{Z^{\prime \prime}}$, as shown in Fig. 2(b). One can see that the $\chi^{2}$ is better in the region with $g_{Z^{\prime \prime}}<0.4$ and $m_{Z^{\prime \prime}}<100 \mathrm{GeV}$, and hence in next section, we study the LHC phenomenology in the favored region.

\section{LHC phenomenology}

The $Z^{\prime \prime}$ boson is produced in collider experiments via a diagram shown in Fig. 3. An important feature of this model is that events with $\mu$ or $\tau$ (but not $e$ ) are affected by the $Z^{\prime \prime}$ production. At the LHC, the production cross section of the $Z^{\prime \prime}$ boson is $O(1)$ fb for $m_{Z^{\prime \prime}}=O(100) \mathrm{GeV}$ and $g_{Z^{\prime \prime}}=O(0.1)$. Therefore, it would be interesting to study whether the $Z^{\prime \prime}$ effects are measurable. In this section, the results based on four signal samples with $m_{Z^{\prime \prime}}=60,80,90$, and $100 \mathrm{GeV}$ are shown. The gauge coupling is fixed as $g_{Z^{\prime \prime}}=0.3$ throughout this section.

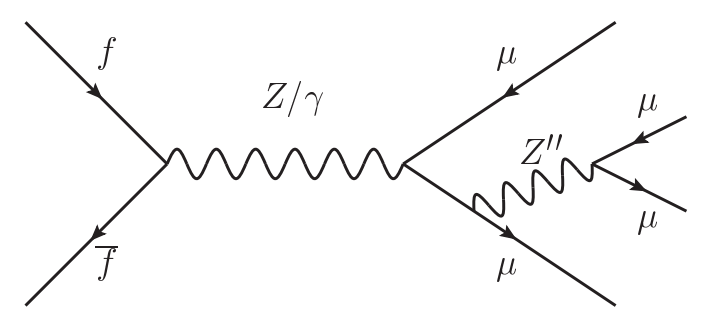

Figure 3: Feynman diagram for a typical $Z^{\prime \prime}$ boson production process at the tree-level.

\section{1 $4 \mu$ channel}

Both CMS [9] and ATLAS [10] have reported the measurements of $\mathrm{Z}$ decays to four leptons at $\sqrt{s}=7$ and $8 \mathrm{TeV}$. Their measurements would be sensitive to the light $Z^{\prime \prime}$ boson. Since the ATLAS result is based on much larger set of data, we adopt the ATLAS analysis. In the ATLAS analysis [10], they search for the production of four leptons, $e^{+} e^{-} e^{+} e^{-}(4 e), \mu^{+} \mu^{-} \mu^{+} \mu^{-}(4 \mu)$, and $e^{+} e^{-} \mu^{+} \mu^{-}(2 e 2 \mu)$ at the $Z$ resonance.

Using the set of selection cuts ATLAS have used, we compare our simulation results with the ATLAS results. In Fig. 4, we show the di-lepton invariant mass $m_{12}$ distribution in the SM (dashed line) and $Z^{\prime \prime}$ models with $m_{Z^{\prime \prime}}=60 \mathrm{GeV}$ (blue line) and $80 \mathrm{GeV}$ (red line). Here the $m_{12}$ is defined by an invariant mass of the same-flavor and opposite-sign di-lepton pair which is the closest to the $Z$ boson mass among the possible combinations, while the other one is called $m_{34}$. The ATLAS data for the integrated luminosities of $4.6 \mathrm{fb}^{-1}$ at $\sqrt{s}=7 \mathrm{TeV}$ and $20.7 \mathrm{fb}^{-1}$ at $\sqrt{s}=8 \mathrm{TeV}$ are also shown. Since a large excess over the SM result is seen around $m_{12} \simeq m_{Z^{\prime \prime}}$ in the $m_{12}$ distribution for the $Z^{\prime \prime}$ model with $m_{Z^{\prime \prime}}=60 \mathrm{GeV}$, we conclude that the $Z^{\prime \prime}$ model with $m_{Z^{\prime \prime}}=60 \mathrm{GeV}$ is excluded by the ATLAS data. ${ }^{1}$

On the other hand, the result for a case with $m_{Z^{\prime \prime}}=80 \mathrm{GeV}$ is almost the same as the one of the SM. Therefore, the current ATLAS analysis is not sensitive to the $Z^{\prime \prime}$ model with $m_{Z^{\prime \prime}}=80 \mathrm{GeV}$.

\footnotetext{
${ }^{1}$ The ALEPH has reported a study of the four fermion final state at the $\mathrm{Z}$ resonance [11], and we have checked that their data put constraints weaker than the current LHC constraints.
} 


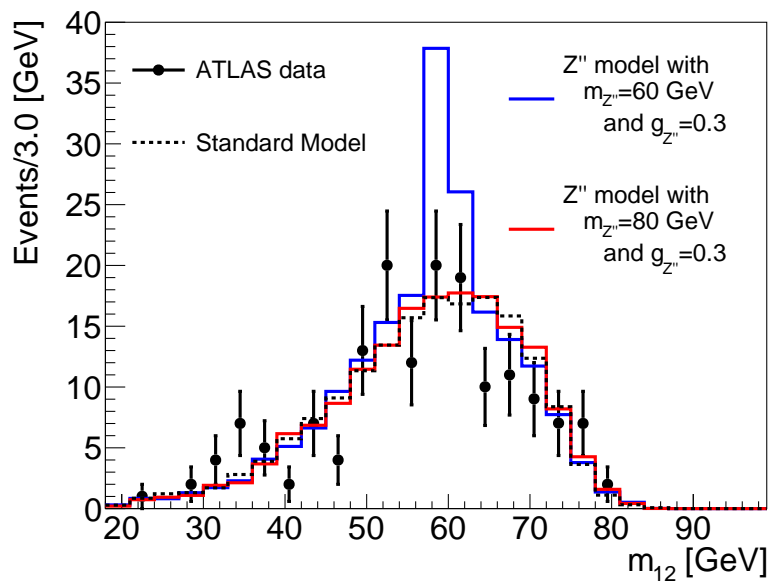

Figure 4: The $m_{12}$ distributions for the SM (dashed) and for the $Z^{\prime \prime}$ models with $m_{Z^{\prime \prime}}=60 \mathrm{GeV}$ (blue) and 80 $\mathrm{GeV}$ (red). All channels ( $4 e, 2 e 2 \mu$ and $4 \mu$ ) are summed up. Combined results for the integrated luminosities of $4.6 \mathrm{fb}^{-1}$ at $\sqrt{s}=7 \mathrm{TeV}$ and $20.7 \mathrm{fb}^{-1}$ at $\sqrt{s}=8 \mathrm{TeV}$ are shown. The figure is taken from Ref. [1].

In order to gain sensitivity for the heavier $Z^{\prime \prime}$ boson, we propose the following optimized selection cuts: (1) $m_{4 \mu}>m_{Z}+10 \mathrm{GeV}$ and reject the Higgs mass region, $\left|m_{4 \mu}-m_{h}\right|>10 \mathrm{GeV}$, (2) $\left|m_{34}-m_{Z}\right|>5 \mathrm{GeV}$ in addition to $p_{T}, \eta$ and $\Delta R$ cuts which the ATLAS have used. Since the signal events are mainly through s-channel off-shell $Z$ boson, we reject the contributions through on-shell $Z$ boson as well as on-shell Higgs boson by the first cut (1). The second cut (2) is for rejecting $Z Z$ production process, which is another SM background, where both $m_{12}$ and $m_{34}$ tend to be close to $m_{Z}$. On the other hand, in the $Z^{\prime \prime}$ signal events, $m_{12}$ tends to be $m_{Z^{\prime \prime}}$, but $m_{34}$ does not have to be close to any particular value. Therefore, it efficiently rejects the $Z Z$ backgrounds while keeping most of the $Z^{\prime \prime}$ signal. In Fig. 5, we show the distribution of the di-muon invariant mass $m_{12}$ in $p p \rightarrow 4 \mu$ in the SM (dashed line) and $Z^{\prime \prime}$ model with (a) $m_{Z^{\prime \prime}}=80 \mathrm{GeV}$, (b) $90 \mathrm{GeV}$, and (c) $100 \mathrm{GeV}$ after imposing the optimized cuts for the integrated luminosities of $4.6 \mathrm{fb}^{-1}$ at $\sqrt{s}=7$ $\mathrm{TeV}$ and $20.7 \mathrm{fb}^{-1}$ at $\sqrt{s}=8 \mathrm{TeV}$. Although small excesses around $m_{12} \simeq m_{Z^{\prime \prime}}$ would be seen from Fig. 5, they are not statistically significant. However, we estimate that the LHC data at $14 \mathrm{TeV}$ run

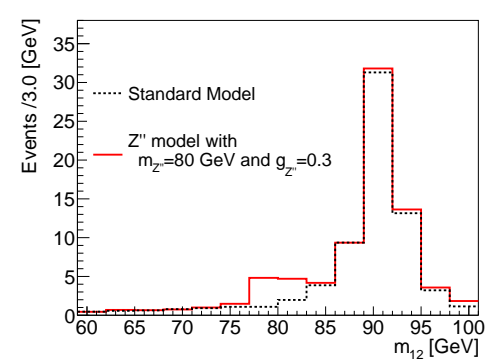

(a)

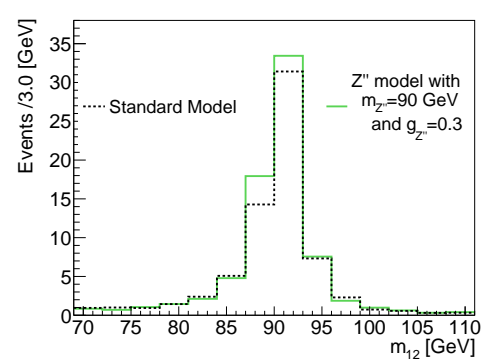

(b)

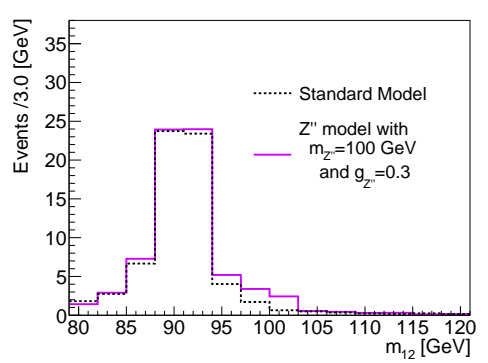

(c)

Figure 5: The distribution of the di-muon invariant mass $m_{12}$ in $p p \rightarrow 4 \mu$ in the SM (dashed line), $Z^{\prime \prime}$ model with $m_{Z^{\prime \prime}}=80,90$, and $100 \mathrm{GeV}$ (solid lines, from left to right) after imposing the optimized cuts. Combined integrated luminosities of $4.6 \mathrm{fb}^{-1}$ at $\sqrt{s}=7 \mathrm{TeV}$ and $20.7 \mathrm{fb}^{-1}$ at $\sqrt{s}=8 \mathrm{TeV}$ are assumed. The figures are taken from Ref. [1]. 
with more luminosities would be enough to observe the clear $Z^{\prime \prime}$ boson signal in $4 \mu$ channel with $m_{Z^{\prime \prime}}<100 \mathrm{GeV}$.

\section{2 $2 \mu 2 \tau$ channel}

The $Z^{\prime \prime}$ boson can couple to not only $\mu$ but also $\tau$. In order to test this feature, it is important to see the pattern of the interactions of the $Z^{\prime \prime}$ boson. One of interesting processes is $2 \mu 2 \tau$ channel. To study this channel, we require the following cuts:

1. two $\tau$ jets exist satisfying $p_{T, \tau}>20 \mathrm{GeV}$ and $\left|\eta_{\tau}\right|<2.3$, only hadronically decaying $\tau$ 's.

2. two oppositely charged muons exist satisfying $p_{T, \mu}>10 \mathrm{GeV}$ and $\left|\eta_{\mu}\right|<2.7$, the two muons are well separated as $\Delta R>0.1$.

3. requiring the invariant mass cut for the two $\tau$ 's, $m_{\tau \tau}>120 \mathrm{GeV}$, where we adopt the collinear approximation for the $\tau$ momentum reconstruction.

We select events with $2 \mu$ and $2 \tau$ by cuts (1) and (2). The 3rd cut (3) rejects the SM ZZ backgrounds because the signal is not enhanced at $m_{\tau \tau} \sim m_{Z}$ nor $m_{Z^{\prime \prime}}$ once we require $m_{\mu \mu} \sim m_{Z^{\prime \prime}}$, while both $m_{\mu \mu}$ and $m_{\tau \tau}$ are enhanced at $m_{Z}$ in the SM ZZ background.

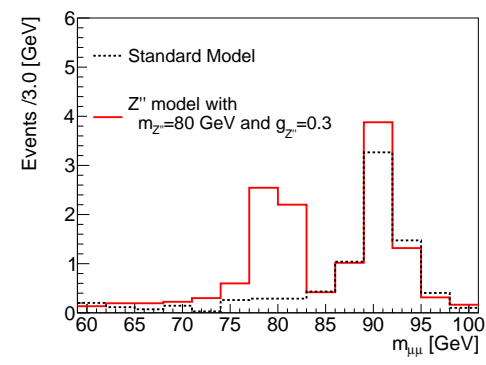

(a)

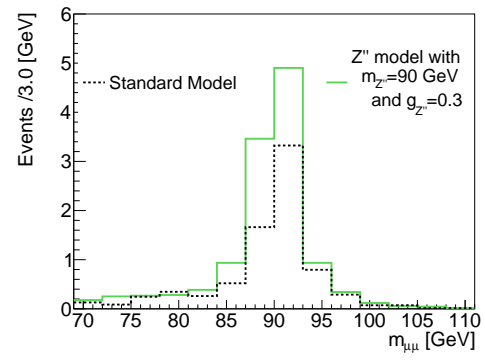

(b)

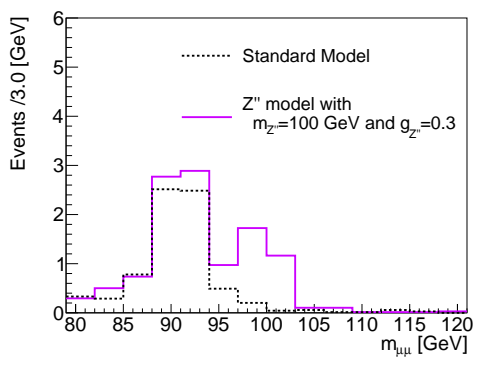

(c)

Figure 6: The $\left(m_{\mu \mu}\right)$ distributions in the $2 \mu 2 \tau$ channel at $\sqrt{s}=14 \mathrm{TeV}$ for the SM (dashed line) and for the $Z^{\prime \prime}$ model with $m_{Z^{\prime \prime}}=80,90$, and $100 \mathrm{GeV}$ (solid lines, from left to right). The integrated luminosity of 300 $\mathrm{fb}^{-1}$ is assumed. The figures are taken from Ref. [1].

In Fig. 6, we show the di-muon invariant mass $m_{\mu \mu}$ distributions for the SM (dashed line) and $Z^{\prime \prime}$ model with $m_{Z^{\prime \prime}}=$ (a) $80 \mathrm{GeV}$, (b) $90 \mathrm{GeV}$, and (c) $100 \mathrm{GeV}$ (solid lines), respectively for the integrated luminosities of $300 \mathrm{fb}^{-1}$ at $\sqrt{s}=14 \mathrm{TeV}$. Although the number of signal events is small, excesses are seen in the signal region $m_{\mu \mu} \simeq m_{Z^{\prime \prime}}$ in the $Z^{\prime \prime}$ models. More data the high luminosity LHC would strengthen the signal observation. We estimate that the luminosities needed for discovery are $500 \mathrm{fb}^{-1}, 2900 \mathrm{fb}^{-1}$, and $730 \mathrm{fb}^{-1}$ for $m_{Z^{\prime \prime}}=80,90$, and $100 \mathrm{GeV}$, respectively.

\section{Summary}

New particles with the mass of the order of the EW scale with a significant coupling to the muon sector can accommodate the muon $\mathrm{g}-2$ anomaly. The LHC would be an important experiment to probe the new physics origin of the muon g-2 anomaly because of the high luminosity and cleanness of the muon signature. 
In this paper, we consider the $L_{\mu}-L_{\tau}$ gauge symmetric model as one of possibilities to explain the muon g-2 anomaly. We have identified the parameter space which is consistent with the EW precision measurements as well as the muon g-2. Since the region with $m_{Z^{\prime \prime}}<100 \mathrm{GeV}$ and $g_{Z^{\prime \prime}}<0.4$ is favored, the LHC has a great potential to probe the model. We have shown that not only $4 \mu$ channel but also $2 \mu 2 \tau$ channel at $14 \mathrm{TeV}$ LHC run would probe the important parameter space of the $Z^{\prime \prime}$ model. Future LHC data are crucial to test the new physics models responsible for the muon g-2 anomaly.

\section{References}

[1] K. Harigaya, T. Igari, M. M. Nojiri, M. Takeuchi and K. Tobe, arXiv:1311.0870 [hep-ph].

[2] J. Beringer et al. [Particle Data Group Collaboration], Phys. Rev. D 86, 010001 (2012) and 2013 partial update for the 2014 edition.

[3] K. Hagiwara, R. Liao, A. D. Martin, D. Nomura and T. Teubner, J. Phys. G 38, 085003 (2011); T. Teubner, K. Hagiwara, R. Liao, A. D. Martin and D. Nomura, Chin. Phys. C 34, 728 (2010); M. Benayoun, P. David, L. DelBuono and F. Jegerlehner, Eur. Phys. J. C 72, 1848 (2012); F. Jegerlehner and R. Szafron, Eur. Phys. J. C 71, 1632 (2011); F. Jegerlehner and A. Nyffeler, Phys. Rept. 477, 1 (2009); M. Davier, A. Hoecker, B. Malaescu and Z. Zhang, Eur. Phys. J. C 71, 1515 (2011) [Erratum-ibid. C 72, 1874 (2012)].

[4] A. Czarnecki, W. J. Marciano and A. Vainshtein, Phys. Rev. D 67, 073006 (2003) [Erratum-ibid. D 73, 119901 (2006)]; C. Gnendiger, D. Stöckinger and H. Stöckinger-Kim, Phys. Rev. D 88, 053005 (2013).

[5] T. Moroi, Phys. Rev. D 53, 6565 (1996) [Erratum-ibid. D 56, 4424 (1997)].

[6] For example, see T. Hambye, K. Kannike, E. Ma and M. Raidal, Phys. Rev. D 75, 095003 (2007); S. Kanemitsu and K. Tobe, Phys. Rev. D 86, 095025 (2012).

[7] P. Fayet, Phys. Rev. D 75, 115017 (2007); M. Pospelov, Phys. Rev. D 80, 095002 (2009); S. N. Gninenko and N. V. Krasnikov, Phys. Lett. B 513, 119 (2001); M. Endo, K. Hamaguchi and G. Mishima, Phys. Rev. D 86, 095029 (2012); H. Davoudiasl, H. -S. Lee and W. J. Marciano, Phys. Rev. D 86, 095009 (2012).

[8] X. -G. He, G. C. Joshi, H. Lew and R. R. Volkas, Phys. Rev. D 44, 2118 (1991); S. Baek, N. G. Deshpande, X. G. He and P. Ko, Phys. Rev. D 64, 055006 (2001); E. Ma, D. P. Roy and S. Roy, Phys. Lett. B 525, 101 (2002); S. Baek and P. Ko, JCAP 0910, 011 (2009); E. Salvioni, A. Strumia, G. Villadoro and F. Zwirner, JHEP 1003, 010 (2010); J. Heeck and W. Rodejohann, Phys. Rev. D 84, 075007 (2011); W. -Z. Feng, P. Nath and G. Peim, Phys. Rev. D 85, 115016 (2012).

[9] S. Chatrchyan et al. [CMS Collaboration], JHEP 1212, 034 (2012) [arXiv:1210.3844 [hep-ex]].

[10] ATLAS Collaboration, ATLAS-CONF-2013-055 (May 27, 2013).

[11] D. Buskulic et al. [ALEPH Collaboration], Z. Phys. C 66, 3 (1995). 